\title{
Delayed Consensus Problem for Single and Double Integrator Systems
}

\author{
Martín Velasco-Villa, ${ }^{1}$ Josué Heras-Godínez, ${ }^{1}$ \\ José Alejandro Vázquez-Santacruz, ${ }^{2}$ and Varinia Fragoso-Rubio ${ }^{1}$ \\ ${ }^{1}$ Departamento de Ingeniería Eléctrica, Sección de Mecatrónica, Centro de Investigación y de Estudios Avanzados del IPN, \\ Avenida I.P.N. No. 2508, Col. San Pedro Zacatenco, 07360 México, DF, Mexico \\ ${ }^{2}$ Facultad de Ingeniería, Universidad Veracruzana, Calzada Adolfo Ruiz Cortines s/n, Fracc. Costa Verde, \\ 94294 Boca del Río, VER, Mexico
}

Correspondence should be addressed to Josué Heras-Godínez; jheras@cinvestav.mx

Received 18 December 2014; Revised 16 March 2015; Accepted 18 March 2015

Academic Editor: Guangming Xie

Copyright (C) 2015 Martín Velasco-Villa et al. This is an open access article distributed under the Creative Commons Attribution License, which permits unrestricted use, distribution, and reproduction in any medium, provided the original work is properly cited.

\begin{abstract}
This work deals with the analysis of the consensus problem for networks of agents constituted by single and double integrator systems. It is assumed that the communication among agents is affected by a constant time-delay. Previous and numerous analysis of the problem shows that the maximum communication time-delay that can be introduced to the network without affecting the consensus of the group of the agents depends on the considered topology. In this work, a control scheme that is based on the estimation of future states of the agents and that allows increasing the magnitude of a possible time-delay affecting the communication channels is proposed. How the proposed delay compensation strategy is independent of the network topology in the sense that the maximum allowable time-delay that could be supported by the network depends on a design parameter and not on the maximum eigenvalue of the corresponding Laplacian matrix is shown. It is formally proven that, under the proposed prediction scheme, the consensus of the group can be achieved by improving the maximum time-delay bounds previously reported in the literature. Numerical simulations show the effectiveness of the proposed solution.
\end{abstract}

\section{Introduction}

In the last years, the study of multiagent systems has deserved an increasing attention of the control community. These systems have been involved in many practical applications in everyday life and industry, as the case of the formation problem of unnamed aerial vehicles (UAV) [1], the control of a group of satellites [2], or formation control vehicles [35]. Some other applications are related to distributed sensors; automatic vehicular control; or analysis of communication networks $[6,7]$.

A common problem in the synchronization of multiagent systems is the consensus problem [8], which is referred to the control and coordination of a set of agents within a communication network. In [3], the importance of the Laplacian of the network in the solution of the consensus problem for a set of agents with linear dynamics is shown.
The problem has also been addressed in [8-11] getting the asymptotic convergence of the agent into the set.

In an attempt to achieve consensus, an additional challenge arises when the agents coordination involves timedelays in the communication network which governs the agents. In control systems, time-delays frequently appear due to phenomena as material transportation, recycle loops, or even the approximation of high order systems by means of reduced order dynamics [12]. Several solutions have been proposed when dealing with stable processes with time-delay; in the linear case, when the time-delay is located at the input or output of the system, a common practice consists on eliminating the effect of the delayed signal by means of an adequate feedback [13-15].

In general, the consensus problem has been commonly treated by considering that the agents are free of delays. The problem has been properly described in $[7,16,17]$ and 
a solution has been clearly stated. The consensus problem has been also extended to the case of bounded inputs in [18]. On the other hand, the consideration of time-delay has been studied under several communication schemes, for example, by assuming that the agents are single integrators and that the time-delay is constant. Under these circumstances, an initial analysis that states the maximum time-delay that can be supported by the network is given in [9]. Another scheme, proposed in [19], assumes that the delays are induced only by the neighbor agents and that the local variables are free of delay. In [20], the problem is analyzed by considering that the delay exists on the position variables but not in the velocity of the neighbor agents; the same conditions are imposed in [21] where the velocity error is not used on the control law. By considering the local variables of the agents free of delays, in [22], the problem is studied in a discrete-time context, as well as [23-25]. A delayed position and velocity feedback is considered in [16], while the consensus problem is analyzed in [26] under the assumption of fixed topologies and in [27] with both fixed and switched topologies.

Specific techniques are used to prove stability of a set of agents, for example, a frequency approach has been addressed in $[15,28,29]$ by means of the Nyquist criterion, even for the presence of disturbance and noise as in [23, 30]. Also, the problem is not only addressed by considering a constant delay, but the time varying delay is also considered in works as $[27,31]$. In addition, some strategies based on artificial neural networks are addressed in [32].

In particular, the protocol adopted in [9], for the case of single integrator agents, guarantees the consensus, with their states asymptotically converging to the average of the initial conditions, instead of an arbitrary constant. The authors explicitly provide the largest time-delay that can be applied to the network without breaking the consensus. It is also shown that this largest delay is inversely proportional to the maximum eigenvalue of the Laplacian matrix induced by the network. In the case of double integrator agents with timedelays, in $[21,22,26]$, it is also shown that the solution of the problem is related with the largest eigenvalue of the Laplacian matrix of the network.

By considering the induced restriction by the Laplacian matrix eigenvalues to the solution of the delayed consensus problem, the objective of this work is to increase the largest time-delay that allows the solution of the problem. With this aim, a prediction-observer scheme that provides estimated future states of the agents that are used in the design of an adequate feedback is proposed, inspired by the solution of the free delay case originally given in [16].

The rest of the work is organized as follows. In Section 2, a brief recall of the graph theory and the Laplacian matrix is presented. In Section 3, the consensus problem is stated referring to the previous works reported in the literature. The single integrator case is addressed in Section 4 and the obtained result is generalized to the double integrator case in Section 5. Numerical simulations are presented in Section 6, showing the effectiveness of the proposed control scheme. Finally, some conclusions are given in Section 7.

\section{Graph Theory and Laplacian Matrix}

Graph theory studies structures of vertex, nodes, or point sets and edge or line sets $[8,33,34]$. Being of interest for this work, in what follows a summary of this topic is presented.

2.1. Graph Classes and Definitions. Let $G=\{N, E\}$ be an ordered graph where

(i) $N$ is a nonempty node set defined as $N=\{1, \ldots, n\}$;

(ii) $E$ is an edge set of ordered node pairs, $E \subseteq N \times N$;

(iii) an edge from $G$ is denoted as $e_{i j}=(i, j)$;

(iv) the grade $N$ of a node equals the number of edges $e_{i j}$ that belong to the node.

A directed graph is a pair $(N, E)$ where the edge $(i, j) \in E$ denotes that a $j$-agent can obtain information from an $i$-agent but not necessarily in the opposite way.

For the edge $(i, j), i$ is the parent node and $j$ is the child one. In contrast to a directed graph, a pair of nodes in an undirected graph is unordered; that is, the edge $(i, j)$ denotes that agents $i$ and $j$ can obtain information from each other. A directed path is a sequence of edges in a directed graph of the form $\left(i_{1}, i_{2}\right),\left(i_{2}, i_{3}\right), \ldots$. An undirected path in an undirected graph is defined analogously. A cycle is a directed path that starts and ends at the same node. A directed graph is strongly connected if there is a directed path from a node to any other node. An undirected graph is connected if there is an undirected path between every pair of distinct nodes. The adjacency matrix $A=\left[a_{i j}\right] \in \mathbb{R}^{n \times n}$ of a directed graph with node set $N=\{1, \ldots, n\}$ is defined such that the weight $a_{i j}$ is positive if $(i, j) \in E$ and $a_{i j}=0$ if $(i, j) \notin E$. If the weights are not relevant, then $a_{i j}$ is set equal to 1 for all $(i, j) \in E$. A graph is balanced if $\sum_{j=1}^{n} a_{i j}=\sum_{j=1}^{n} a_{j i}$ for all $i$. For an undirected graph, $A$ is symmetric, and thus every undirected graph is balanced.

2.2. Laplacian Matrix. The Laplacian matrix $L=\left[l_{i j}\right] \in \mathbb{R}^{n \times n}$ of a directed graph is given by $l_{i i}=\sum_{j \neq i} a_{i j}$ and $l_{i j}=-a_{i j}$ for all $i \neq j$.

\subsubsection{Laplacian Matrix Properties}

(i) $l_{i j} \leq 0, i \neq j$

(ii) The sum of the elements of each row is zero; Consider $\sum_{j=1}^{n} l_{i j}=0, i=1, \ldots, n$.

(iii) For an undirected graph, $L$ is symmetric.

(iv) For directed and undirected graphs, 0 is an eigenvalue of $L$, the sum of the elements of each row is zero, and it has an eigenvector $1_{n}=[1, \ldots, 1]^{T}$, of dimension $n \times 1$, corresponding to the zero eigenvalue.

(v) $L$ is diagonally dominant and has no negative entries on its diagonal.

(vi) All nonzero eigenvalues of $L$ are positive, while, in a directed graph, all nonzero eigenvalues of $L$ have positive real parts. Therefore, all nonzero eigenvalues of $-L$ have negative real parts. 
(vii) Zero is always an eigenvalue of $L$ with at least multiplicity one.

(viii) For an undirected graph, zero is a simple eigenvalue of $L$ if and only if the undirected path is connected.

(ix) For a directed graph, zero is a simple eigenvalue of $L$ if and only if the graph has a spanning tree.

(x) If the $i$ th node of $G$ is disconnected, then $l_{i j}=l_{j i}=0$ for all $j$.

\section{Statement of the Problem}

The main idea of the consensus protocol is to impose a similar dynamic to the state of each agent. If the communication channels in the network allow continuous flow information, then the evolution of each agent can be modeled, for instance, by means of a differential equation. In this work it is considered fixed information exchange topologies with Laplacian matrices with a single zero eigenvalue; that is, directed graphs with an spanning tree or undirected connected graphs [34] are considered.

Definition 1. The consensus problem is solved for a group of agents if, for all initial condition $x_{i}(0)$, it is satisfied as follows:

$$
\left\|x_{i}(t)-x_{j}(t)\right\| \longrightarrow 0
$$

as $t \rightarrow \infty$, for all $i, j=1, \ldots, n$.

3.1. Consensus for Single Integrator Agents. Consider the class of single-input single-output agents of the form

$$
\dot{x}_{i}(t)=u_{i}(t) \text {, }
$$

for $i=1,2, \ldots, n$, with $x_{i} \in \mathbb{R}$ being the state associated with the $i$ th agent while $u_{i} \in \mathbb{R}$ is the corresponding input signal. Define, for a group of $n$ agents, $x=\left[x_{1}, \ldots, x_{n}\right]^{T} \in \mathbb{R}^{n}$ and $u=\left[u_{1}, \ldots, u_{n}\right]^{T} \in \mathbb{R}^{n}$.

The consensus protocol for the case of a fixed $[4,8,9]$ or variant [9] topology in the case of agents (2) is given by

$$
u_{i}(t)=-\sum_{j=1}^{n} a_{i j}\left(x_{i}(t)-x_{j}(t)\right) .
$$

The closed-loop (2)-(3) produces

$$
\dot{x}(t)=-L x(t),
$$

where $L \in \mathbb{R}^{n \times n}$ is the Laplacian matrix induced by the considered topology.

In this work, it will be assumed that, for a fixed topology of a directed graph with a spanning tree $G=\{N, E\}$, there exists a constant time-delay $\tau_{i j}>0$ affecting the edge $e_{i j} \in E$; in particular, it will be considered that the time-delay is equal for all the communication channels: $\tau_{i j}=\tau$. Under the effects of communication time-delays, the consensus protocol (3) is modified to the delayed state causal feedback [9]:

$$
u_{i}(t)=-\sum_{j=1}^{n} a_{i j}\left(x_{i}(t-\tau)-x_{j}(t-\tau)\right)
$$

that produces the closed-loop system:

$$
\dot{x}(t)=-L x(t-\tau),
$$

where $x \in \mathbb{R}^{n}$ and $x_{i}(t-\tau)$ is the state of the $i$ th agent delayed $\tau$ units of time.

Remark 2. It should be noticed that, under the assumption that $\tau_{i j}=\tau_{1}>0$, the delayed consensus problem can be stated by considering a set of delayed agents:

$$
\dot{x}_{i}(t)=u_{i}\left(t-\tau_{1}\right)
$$

with a consensus protocol given in (3). In this form, agents (7), explicitly describe the effects of the communication channels. Notice also that, in the case that protocol (5) is applied to agents (7), a closed-loop system is obtained in the form

$$
\dot{x}(t)=-L x\left(t-\tau_{2}\right),
$$

for $\tau_{2}=\tau+\tau_{1}$. Therefore, the solution for the delayed consensus problem is equivalent for system (6) or (8).

Based on the previous remark, the consensus problem will be stated in this work for a set of delayed agents

$$
\dot{x}_{i}(t)=u_{i}(t-\tau) \text {. }
$$

By considering a fixed topology, undirected and connected graphs, the consensus problem for agents (2) and protocol (5) (equivalently, agents (9) and protocol (3)) is solved, if and only if [9]

$$
0 \leq \tau<\frac{\pi}{2 \sigma_{\max }(L)}
$$

where $L$ is the Laplacian of the communication graph and $\sigma_{\max }(L)$ is the largest eigenvalue of $L$.

3.2. Consensus for Double Integrator Agents. As in the case of integrator agents, it will be assumed that there exist constant time-delays $\tau_{i j}=\tau$ (corresponding to the edge $e_{i j} \in E$ ) affecting the communications channels. According to Remark 2, the effects of communication time-delays can be included on the agent dynamics in the form

$$
\begin{aligned}
& \dot{x}_{i_{1}}(t)=x_{i_{2}}(t), \\
& \dot{x}_{i_{2}}(t)=u_{i}(t-\tau),
\end{aligned}
$$

for $i=1,2, \ldots, n$ with $x_{i_{1}}, x_{i_{2}} \in \mathbb{R}$ being the state of the $i$ th agent and $u_{i} \in \mathbb{R}$ the corresponding input signal. $\tau>0$ is the time-delay affecting the $i$ th input signal.

The consensus for system (11) is achieved according to Definition 1; that is, $x_{i_{1}}(t) \rightarrow x_{j_{1}}(t)$ and $x_{i_{2}}(t) \rightarrow x_{j_{2}}(t)$ when $t \rightarrow \infty$, for all $x_{i_{1}}(0)$ and $x_{i_{2}}(0)$.

System (11) can be rewritten as

$$
\begin{gathered}
\dot{x}_{1}(t)=x_{2}(t), \\
\dot{x}_{2}(t)=u(t-\tau),
\end{gathered}
$$


where $x_{1}=\left[\begin{array}{lll}x_{1_{1}} & \cdots & x_{n_{1}}\end{array}\right]^{T}, x_{2}=\left[\begin{array}{lll}x_{1_{2}} & \cdots & x_{n_{2}}\end{array}\right]^{T}$, and $u=$ $\left[\begin{array}{lll}u_{1} & \cdots & u_{n}\end{array}\right]^{T}$.

For the free delay case, $\tau=0$ in (11), the solution of the consensus problem in the case of a directed graph containing a spanning tree is obtained by means of the state feedback $[7,18]$,

$$
u_{i}=-\sum_{j=1}^{n} a_{i j}\left[\left(x_{i_{1}}-x_{j_{1}}\right)+\gamma\left(x_{i_{2}}-x_{j_{2}}\right)\right],
$$

for sufficiently large positive $\gamma \in \mathbb{R}$, producing a closed-loop system with exactly two zero poles and all the remaining poles with negative real part.

For a fixed undirected topology having a spanning tree, in [26], a solution to the delayed consensus problem (in the case that $\tau_{i j}=\tau$ ) for double integrator agents is presented by considering a modification of the causal feedback (13) in the form

$$
\begin{aligned}
u_{i}(t)=-\sum_{j=1}^{n} a_{i j}[ & k_{1}\left(x_{i_{1}}(t)-x_{j_{1}}(t)\right) \\
& \left.+k_{2}\left(x_{i_{2}}(t)-x_{j_{2}}(t)\right)\right],
\end{aligned}
$$

with $k_{1}$ and $k_{2}$ as positive constants. This solution is stated for a maximum time-delay $\tau^{*}$ such that $\tau<\tau^{*}$, with

$$
\tau^{*} \leq \frac{k_{2} \arctan (\sqrt{\zeta})(1-\zeta)}{k_{1} \sqrt{\zeta}}
$$

where $\zeta$ is the positive root of

$$
\begin{aligned}
\zeta^{3}+ & \left(1+\frac{4 k_{1}}{k_{2}^{2} \sigma_{\max }(L)}\right) \zeta^{2} \\
& +\left(\frac{4 k_{1}}{k_{2}^{2} \sigma_{\max }(L)}-1\right) \zeta-1=0 .
\end{aligned}
$$

It is important to point out that the existing solutions for the delayed consensus problem for single (9) and double integrators (11) are based on the use of causal feedbacks of the forms (3) and (13) synthesized at actual time $t$ producing time-delayed closed-loop dynamics.

Suppose now that protocols (3) and (13) can be designed in terms of the future value, $\tau$ units of time, of the agents states, in the form

$$
u_{i}(t)=-\sum_{j=1}^{n} a_{i j}\left(x_{i}(t+\tau)-x_{j}(t+\tau)\right)
$$

for the single integrator case and

$$
\begin{aligned}
u_{i}(t)=-\sum_{j=0}^{n} a_{i j}\left[\left(x_{i_{1}}(t+\tau)-x_{j_{1}}(t+\tau)\right)\right. & \\
& \left.+\gamma\left(x_{i_{2}}(t+\tau)-x_{j_{2}}(t+\tau)\right)\right]
\end{aligned}
$$

for the double integrator agents.
It is easy to see that protocols (17) and (18) would solve the delayed consensus problem producing closed-loop systems free of time-delay for the single and double integrator cases. Notice that the convergence properties in the case of system (9)-(17) or (11)-(18) would be equivalent to the ones of the free-delay cases [4, 7-9]. It should be highlighted that protocols (17) and (18) require future values of the agents states that prevent their practical implementation due to their noncausal nature.

The main objective of this work is to propose estimated state protocols of the forms (17) and (18) where instead of the actual future states $x_{i}(t+\tau)$ and $x_{i_{j}}(t+\tau)$, the use of future estimated states $\hat{x}_{i}(t+\tau)$ and $\widehat{x}_{i_{j}}(t+\tau)$ obtained by an adequate prediction observer is considered.

\section{Prediction-Observer Scheme}

As a solution to the delayed consensus problem, for fixed topologies having a spanning tree, a predictor-observer scheme that provides the future estimation of the state of the agents is designed. The scheme has the advantage that the convergence conditions depend on the design parameters and they are not related to the location of the eigenvalues of the Laplacian matrix as it has been previously reported.

4.1. Prediction-Observer for Single Integrator Agents. To synthesize the predictor-observer based solution, how the considered agents given in (2) can be expressed in terms of future states is shown first. With this aim, consider the new variables

$$
w_{i}(t)=x_{i}(t+\tau)
$$

for $i=1, \ldots, n$ or equivalently

$$
w_{i}(t-\tau)=x_{i}(t)
$$

producing a new state vector $w=\left[w_{1}, w_{2}, \ldots, w_{n}\right]^{T}$ for the group of agents. Taking the time derivative, it is obtained that

$$
\dot{w}_{i}(t-\tau)=\dot{x}_{i}(t)=u_{i}(t-\tau),
$$

which by a forward shift of $\tau$ units of time produces

$$
\dot{w}(t)=u(t) .
$$

System (22) represents an advanced system, free of delays, for the original agents given by (2).

Based on (22), the predictor-observer that will provide the future state of system (2) is defined as

$$
\dot{\widehat{w}}(t)=u(t)+\lambda e_{w}(t-\tau),
$$

where $\lambda>0$ is a design constant parameter, $\widehat{w} \in \mathbb{R}^{n}$ is the estimation of $w(t)=x(t+\tau), u(t)$ is the input signal, and $e_{w}(t-\tau)$ is the estimation error, delayed $\tau$ units of time defined as

$$
e_{w}(t-\tau)=w(t-\tau)-\widehat{w}(t-\tau),
$$

with $e_{w i}(t-\tau)=w_{i}(t-\tau)-\widehat{w}_{i}(t-\tau)$ for $i=1, \ldots, n$. 
Remark 3. Contrary to a standard Luenberger observer, in the predictor-observer (23), it is not possible to use the signal $e_{w}(t)$ since

$$
e_{w}(t)=x(t+\tau)-\widehat{x}(t+\tau)
$$

that requires future values of the state $x(t)$ which are not available.

The convergence of the prediction error $e_{w}(t)$ can be analyzed by considering its time evolution

$$
\dot{e}_{w}(t)=-\lambda e_{w}(t-\tau) .
$$

It is well known that system (26) is not only asymptotically but also exponentially stable, [35]. The exponential stability property can be formally stated as follows.

Definition 4. The trivial solution $e_{w}(t)=0$ of the time-delay system (26) is exponentially stable with decay rate $\alpha$, if there exist constants $\alpha>0, \beta \geq 1$ such that

$$
\left\|e_{w}(t)\right\| \leq \beta \sup _{-\tau \leq \theta \leq 0}\left\{\left\|e_{w}(\theta)\right\|\right\} e^{-\alpha t} .
$$

The convergence of the predictor-observer (23) is formally stated in the next result.

Lemma 5. Consider a group of $n$ single integrator agents with a fixed topology having a spanning tree as given in (9) and the predictor-observer defined in (23). Then, for a positive constant $\lambda$, signal $\widehat{w}_{i}(t)$ exponentially converges to the future value $x_{i}(t+$ $\tau)$ for $i=1, \ldots, n$ if and only if

$$
\tau<\frac{\pi}{2 \lambda} .
$$

Proof. The convergence of $\widehat{w}_{i}(t)$ can be established by analyzing the stability properties of the prediction error dynamic (26) by noticing that system (26) can be decomposed into the decoupled scalar systems

$$
\dot{e}_{w i}(t)=-\lambda e_{w i}(t-\tau)
$$

The results presented in [36] can be used to show that system (26) will be stable for all $\tau<\tau^{*}$, if and only if,

$$
\tau^{*}=\frac{\pi}{2 \lambda}
$$

In addition, to get an exponential decay rate for system (26), consider the change of variable

$$
z(t)=e^{\alpha t} e_{w}(t),
$$

with $z=\left[z_{1}, \ldots, z_{n}\right] \in \mathbb{R}^{n}, \alpha>0$. Therefore,

$$
\begin{aligned}
\dot{z}(t) & =\alpha e^{\alpha t} e_{w}(t)+e^{\alpha t} \dot{e}_{w}(t) \\
& =\alpha z(t)+e^{\alpha t}\left(-\lambda e_{w}(t-\tau)\right) \\
& =\alpha z(t)-\lambda e^{\alpha t} e^{-\alpha(t-\tau)} z(t-\tau)
\end{aligned}
$$

yielding

$$
\dot{z}(t)=\alpha z(t)-\lambda e^{\alpha \tau} z(t-\tau)
$$

that produces the decoupled set of differential-difference equations

$$
\dot{z}_{i}(t)=\alpha z_{i}(t)-\lambda e^{\alpha \tau} z_{i}(t-\tau)
$$

for $i=1, \ldots, n$.

From a direct analysis of the characteristic equation of (34) (see [36, page 119]), it is possible to determine that system (33) will be stable for all $\tau<\tau^{*}$ such that

$$
\tau^{*}=\frac{\arccos \left(\alpha / \lambda e^{\alpha t}\right)}{\sqrt{\left(\lambda e^{\alpha \tau}\right)^{2}-\alpha^{2}}} .
$$

In particular, by assuming that the decay rate $\alpha$ in (31) tends to zero, implying that $\lim _{\alpha \rightarrow 0} e^{\alpha t}=1$, it is obtained that

$$
\begin{aligned}
\lim _{\alpha \rightarrow 0} \tau^{*} & =\lim _{\alpha \rightarrow 0} \frac{\arccos (\alpha / \lambda)}{\sqrt{\lambda^{2}-\alpha^{2}}} \\
& =\frac{\arccos (0)}{\lambda} .
\end{aligned}
$$

Since $\arccos (0)= \pm(2 m-1) \pi / 2$ for $m=1,2, \ldots$, considering that $\tau<\tau^{*}$ and that $\tau>0$, the following is obtained:

$$
\lim _{\alpha \rightarrow 0} \tau^{*}=\frac{\pi}{2 \lambda} .
$$

Therefore, since system (33) is stable, the solution of (26) is exponentially stable.

Remark 6. The exponential stability of (26) can also be established from [36, Corollary 5.10, page 240]. In this case, the exponential stability is stated for all time-delay $\bar{\tau}$, such that

$$
\bar{\tau}<\frac{1}{\lambda}
$$

with decay rate $\sigma / \bar{\tau}, \sigma>0$ that satisfies the transcendence equation

$$
-\lambda+\frac{\sigma}{\tau}+\bar{\tau} \lambda^{2} e^{2 \sigma /(1-\alpha)}=0 .
$$

In this case, the obtained time-delay bound is more conservative.

4.2. Prediction-Observer for Double Integrator Agents. In the case of double integrator agents of the form (11), an equivalent system that describes the future dynamic of the state can be obtained by considering the new variables

$$
w_{i 1}(t)=x_{i_{1}}(t+\tau), \quad w_{i_{2}}(t)=x_{i_{2}}(t+\tau),
$$

for $i=1, \ldots, n$. Under these conditions,

$$
\begin{gathered}
\dot{w}_{i 1}(t)=\dot{x}_{i_{1}}(t+\tau)=x_{i_{2}}(t+\tau)=w_{i_{2}}(t), \\
\dot{w}_{i 2}(t)=\dot{x}_{i_{2}}(t+\tau)=u_{i}(t)
\end{gathered}
$$


that is,

$$
\begin{aligned}
& \dot{w}_{i 1}(t)=w_{i 2}(t), \\
& \dot{w}_{i 2}(t)=u_{i}(t) .
\end{aligned}
$$

Defining the estimation errors as

$$
\begin{aligned}
& e_{w_{i 1}}(t)=w_{i 1}(t)-\widehat{w}_{i 1}(t), \\
& e_{w_{i 2}}(t)=w_{i 2}(t)-\widehat{w}_{i_{2}}(t),
\end{aligned}
$$

for $i=1, \ldots, n$, the predictor-observer for system (11) is proposed as

$$
\begin{gathered}
\dot{\widehat{\hat{w}}}_{i 1}(t)=\widehat{w}_{i 2}(t)+\lambda_{i 1} e_{w_{i 1}}(t-\tau), \\
\dot{\widehat{w}}_{i 2}(t)=u_{i}(t)+\lambda_{i 0} e_{w_{i 1}}(t-\tau) .
\end{gathered}
$$

where $\lambda_{i 0}$ and $\lambda_{i 1}$ are positive design constant.

Lemma 7. Consider the group of $n$ double integrator agents (11) with fixed topology having a spanning tree and the predictor-observer (44). Then the estimation signals $\widehat{w}_{i 1}(t)$ and $\widehat{w}_{i 2}(t)$ exponentially converge, respectively, to the future values $x_{i_{1}}(t+\tau)$ and $x_{i_{2}}(t+\tau)$ for $i=1, \ldots, n$ if and only if $\tau<\tau^{*}$ such that

$$
\tau^{*}=\frac{1}{\omega} \arctan \left(\frac{\lambda_{i 1} \omega}{\lambda_{i 0}}\right)
$$

where

$$
\omega=\sqrt{\frac{1}{2} \lambda_{i 1}^{2}+\frac{1}{2} \sqrt{4 \lambda_{i 0}^{2}+\lambda_{i 1}^{4}}}
$$

for positive design constant gains $\lambda_{i 0}$ and $\lambda_{i 1}$.

Proof. Consider the time derivative of the estimation errors (43):

$$
\begin{aligned}
\dot{e}_{w_{i 1}}(t) & =\dot{w}_{i 1}(t)-\dot{\widehat{w}}_{i 1}(t) \\
& =w_{i 2}(t)-\widehat{w}_{i 2}(t)-\lambda_{i 1} e_{w_{i 1}}(t-\tau) \\
& =e_{w_{i 2}}(t)-\lambda_{i 1} e_{w_{i 1}}(t-\tau) ; \\
\dot{e}_{w_{i 2}}(t) & =\dot{w}_{i 2}(t)-\dot{\widehat{w}}_{i 2}(t) \\
& =u_{i}(t)-u_{i}(t)-\lambda_{i 0} e_{w_{i}}(t-\tau) \\
& =-\lambda_{i 0} e_{w_{i}}(t-\tau),
\end{aligned}
$$

equivalently,

$$
\begin{aligned}
& \dot{e}_{w_{i 1}}(t)=e_{w_{i 2}}(t)-\lambda_{i 1} e_{w_{i 1}}(t-\tau), \\
& \dot{e}_{w_{i 2}}(t)=-\lambda_{i 0} e_{w_{i 1}}(t-\tau),
\end{aligned}
$$

for $i=1, \ldots, n$. From (48) it is obtained that

$$
\begin{aligned}
\ddot{e}_{w_{i 1}}(t) & =\dot{e}_{w_{i 2}}-\lambda_{i 1} \dot{e}_{w_{i 1}}(t-\tau) \\
& =-\lambda_{i 0} e_{w_{i 1}}(t-\tau)-\lambda_{i 1} \dot{e}_{w_{i 1}}(t-\tau) ;
\end{aligned}
$$

equivalently,

$$
\ddot{e}_{w_{i 1}}(t)+\lambda_{i 1} \dot{e}_{w_{i 1}}(t-\tau)+\lambda_{i 0} e_{w_{i 1}}(t-\tau)=0 .
$$

The stability of system (50) can be stated by a direct analysis of its characteristic equation [36]:

$$
s^{2}+\left(\lambda_{i 1} s+\lambda_{i 0}\right) e^{-\tau s}=0
$$

By considering that the crossing of the roots of (51) with the imaginary axis occurs for $s=j \omega$, it is obtained that

$$
-\omega^{2}+\left(\lambda_{i 0}+j \lambda_{i 1} \omega\right)(\cos (\omega \tau)-j \sin (\omega \tau))=0 ;
$$

that is,

$$
\begin{aligned}
-\omega^{2} & +\lambda_{i 0} \cos (\omega \tau)-j \lambda_{i 0} \sin (\omega \tau) \\
& +j \lambda_{i 1} \omega \cos (\omega \tau)+\lambda_{i 1} \omega \sin (\omega \tau)=0
\end{aligned}
$$

therefore,

$$
\begin{gathered}
-\omega^{2}+\lambda_{i 0} \cos (\omega \tau)+\lambda_{i 1} \omega \sin (\omega \tau)=0 \\
-\lambda_{i 0} \sin (\omega \tau)+\lambda_{i 1} \omega \cos (\omega \tau)=0
\end{gathered}
$$

From (54b),

$$
\tau=\frac{1}{\omega} \arctan \left(\frac{\lambda_{i 1} \omega}{\lambda_{i 0}}\right)
$$

which substituted in (54a) produces

$$
\begin{aligned}
& \lambda_{i 0} \cos \left(\arctan \left(\frac{\lambda_{i 1} \omega}{\lambda_{i 0}}\right)\right) \\
& +\lambda_{i 1} \omega \sin \left(\arctan \left(\frac{\lambda_{i 1} \omega}{\lambda_{i 0}}\right)\right)=\omega^{2},
\end{aligned}
$$

from where

$$
\lambda_{i 0} \frac{1}{\sqrt{1+\left(\lambda_{i 1} \omega / \lambda_{i 0}\right)^{2}}}+\lambda_{i 1} \omega \frac{\lambda_{i 1} \omega / \lambda_{i 0}}{\sqrt{1+\left(\lambda_{i 1} \omega / \lambda_{i 0}\right)^{2}}}=\omega^{2} .
$$

Equivalently,

$$
\frac{\lambda_{i 0}^{2}+\lambda_{i 1}^{2} \omega^{2}}{\lambda_{i 0} \omega^{2}}=\sqrt{1+\left(\frac{\lambda_{i 1} \omega}{\lambda_{i 0}}\right)^{2}}
$$

that after some simplifications yields

$$
\omega^{6}+\left(\frac{\lambda_{i 0}^{2}-\lambda_{i 1}^{4}}{\lambda_{i 1}^{2}}\right) \omega^{4}-2 \lambda_{i 0}^{2} \omega^{2}-\frac{\lambda_{i 0}^{4}}{\lambda_{i 1}^{2}}=0
$$

To get a positive solution of (59), consider the auxiliary variable

$$
\zeta=\omega^{2}
$$

Then (59) is rewritten in the form

$$
\left(\zeta+\frac{\lambda_{i 0}^{2}}{\lambda_{i 1}^{2}}\right)\left(\zeta^{2}-\lambda_{i 1}^{2} \zeta-\lambda_{i 0}^{2}\right)=0
$$


with the solutions

$$
\begin{aligned}
& \zeta_{1}=-\frac{\lambda_{i 0}^{2}}{\lambda_{i 1}^{2}}, \\
& \zeta_{2}=\frac{1}{2} \lambda_{i 1}^{2}+\frac{1}{2} \sqrt{4 \lambda_{i 0}^{2}+\lambda_{i 1}^{4}}, \\
& \zeta_{3}=\frac{1}{2} \lambda_{i 1}^{2}-\frac{1}{2} \sqrt{4 \lambda_{i 0}^{2}+\lambda_{i 1}^{4}} .
\end{aligned}
$$

Since $\lambda_{i 0}$ and $\lambda_{i 1}$ are positive constants, it is easy to see that $\zeta_{2}$ is the only positive solution. Equations (55) and $w=\sqrt{\zeta_{2}}$ show the convergence of the estimation signals.

In order to get an exponential decay rate for system (48), consider its equivalent description

$$
\dot{e}_{w_{i}}(t)=A e_{w_{i}}(t)+A_{d} e_{w_{i}}(t-\tau),
$$

where $e_{w_{i}}=\left[e_{w_{i 1}}, e_{w_{i 2}}\right]^{T}$,

$$
A=\left[\begin{array}{ll}
0 & 1 \\
0 & 0
\end{array}\right], \quad A_{d}=\left[\begin{array}{ll}
-\lambda_{i 1} & 0 \\
-\lambda_{i 0} & 0
\end{array}\right],
$$

and the change of variable

$$
\xi_{i}(t)=e^{\alpha t} e_{w_{i}}(t), \quad \alpha>0 .
$$

Then, it is possible to write

$$
e_{w_{i}}(t)=e^{-\alpha t} \xi_{i}(t), \quad e_{w_{i}}(t-\tau)=e^{-\alpha(t-\tau)} \xi_{i}(t-\tau),
$$

and therefore

$$
\begin{aligned}
\dot{\xi}_{i}(t)= & \alpha e^{\alpha t} e_{w_{i}}(t)+e^{\alpha t} \dot{e}_{w_{i}}(t) \\
= & \alpha e^{\alpha t} e_{w_{i}}(t)+e^{\alpha t}\left(A e_{w_{i}}(t)+A_{d} e_{w_{i}}(t-\tau)\right) \\
= & {[A+\alpha I] e^{\alpha t} e_{w_{i}}(t) } \\
& +e^{\alpha t} e^{\alpha(t-\tau)} e^{-\alpha(t-\tau)} A_{d} e_{w_{i}}(t-\tau) \\
= & {[A+\alpha I] e^{\alpha t} e_{w_{i}}(t) } \\
& +e^{\alpha \tau} A_{d} e^{\alpha(t-\tau)} e_{w_{i}}(t-\tau),
\end{aligned}
$$

obtaining

$$
\dot{\xi}_{i}(t)=[A+\alpha I] \xi_{i}(t)+e^{\alpha \tau} A_{d} \xi_{i}(t-\tau) .
$$

Notice that if $\xi_{i}(t)$ is stable, then, by $(65), e_{w_{1}}(t)$ will be exponentially stable with decay rate $\alpha$. Since (63) is stable, then, by means of a continuity argument, the stability of (68) can always be established for a sufficiently small constant $\alpha$.

\section{Solution to the Consensus Problem}

5.1. Single Integrator Agents Case. The solution for the consensus problem proposed in [9] for system (9), given in (3), can be redefined by considering the estimated future states, resulting in

$$
u(t)=-L \widehat{w}(t)=-L \widehat{x}(t+\tau),
$$

producing the closed-loop (9)-(69) as

$$
\begin{aligned}
\dot{x}(t) & =-L \widehat{x}(t), \\
\dot{\hat{w}}(t) & =-L \widehat{w}(t)+\lambda e_{w}(t-\tau) .
\end{aligned}
$$

Therefore, by considering the estimation error (24)

$$
e_{w}(t-\tau)=w(t-\tau)-\widehat{w}(t-\tau)=x(t)-\widehat{x}(t),
$$

system (70) can be rewritten as

$$
\begin{gathered}
\dot{x}(t)=-L x(t)+L e_{w}(t-\tau), \\
\dot{e}_{w}(t)=-\lambda e_{w}(t-\tau),
\end{gathered}
$$

where, as defined previously, $x=\left[x_{1}, \ldots, x_{n}\right]^{T}, e_{w}=$ $\left[e_{w 1}, \ldots, e_{w n}\right]^{T}, \lambda>0$, and $L \in \mathbb{R}^{n \times n}$ is the Laplacian matrix related to the topology of the agents.

By considering a nonsingular matrix $P=\left[v_{1}, \ldots, v_{n}\right] \in$ $\mathbb{R}^{n \times n}$ defined by the generalized eigenvectors $v_{i}$ of matrix $L$, it is possible to define the change of coordinates

$$
x=P \chi, \quad \chi=P^{-1} x,
$$

producing a new representation for (72a) in the form

$$
\dot{\chi}=-J \chi+P^{-1} L e_{w}(t-\tau),
$$

where $J=P^{-1} L P$ is the Jordan canonical form of matrix $L$.

The linear nature of (74) allows writing its solution as

$$
\chi(t)=e^{-J t} \chi(0)+\int_{0}^{t} e^{-J(t-s)} P^{-1} L e_{w}(s-\tau) d s .
$$

From the properties of $L$ given in Section 2, the generalized eigenvector associated with the zero eigenvalue satisfies $v_{1}=1_{n}$; therefore,

$$
J=P^{-1} L P=\left[\begin{array}{cccc}
0 & 0 & 0 & 0 \\
0 & B_{1} & 0 & 0 \\
0 & 0 & \ddots & 0 \\
0 & 0 & 0 & B_{r}
\end{array}\right]=\left[\begin{array}{ll}
0 & 0 \\
0 & \bar{J}
\end{array}\right],
$$

where $B_{i} \in \mathbb{R}^{m_{i} \times m_{i}}$ correspond to Jordan blocks related to the independent eigenvalues $\lambda_{i} \neq 0$, with multiplicity $m_{i}, i=$ $1, \ldots, r$, of matrix $L$. From the structure of $J$ in $(76), 1+m_{1}+$ $\cdots+m_{r}=n$ and $\bar{J} \in \mathbb{R}^{(n-1) \times(n-1)}[37]$.

To get the solution of the consensus problem, note that, in (75), $J P^{-1}=P^{-1} L$ and therefore

$$
\chi(t)=\left[\begin{array}{cc}
1 & 0 \\
0 & e^{-\bar{J} t}
\end{array}\right] \chi(0)
$$

$$
+\int_{0}^{t}\left[\begin{array}{cc}
1 & 0 \\
0 & e^{-\bar{J}(t-s)}
\end{array}\right]\left[\begin{array}{ll}
0 & 0 \\
0 & \bar{J}
\end{array}\right] P^{-1} e_{w}(s-\tau) d s ;
$$


equivalently,

$$
\begin{aligned}
\chi(t)= & {\left[\begin{array}{cc}
1 & 0 \\
0 & e^{-\bar{J} t}
\end{array}\right] \chi(0) } \\
& +\left[\int_{0}^{t} e^{-\bar{J}(t-s)} \bar{J} P^{-1} e_{w}(s-\tau) d s\right] .
\end{aligned}
$$
tems

Equation (78) corresponds to the solution of the subsys-

$$
\begin{aligned}
& \dot{\bar{\chi}}_{1}(t)=0, \\
& \dot{\bar{\chi}}_{2}(t)=\bar{J} \bar{\chi}_{2}(t)+f(t)
\end{aligned}
$$

with $\bar{\chi}_{1}=\chi_{1}, \bar{\chi}_{2}=\left[\begin{array}{lll}\chi_{2} & \cdots & \chi_{n}\end{array}\right]^{T}$, and $f(t)=\bar{J} P^{-1} e_{w}(t-\tau)$. By standard properties of linear systems, notice that since $\lim _{t \rightarrow \infty} f(t)=0$, then, when $t \rightarrow \infty$, the solution of the nonautonomous system (79b) corresponds to its homogeneous solution, and therefore it is possible to assure that

$$
\lim _{t \rightarrow \infty} \int_{0}^{t} e^{-J(t-s)} P^{-1} L e_{w}(s-\tau) d s=0 .
$$

It is clear now from $x(t)=P \chi(t)$ and (78) that

$$
\lim _{t \rightarrow \infty} x(t)=P\left[\begin{array}{lc}
1 & 0 \\
0 & 0_{(n-1) \times(n-1)}
\end{array}\right] \chi(0)=P\left[\begin{array}{c}
\chi_{1}(0) \\
0 \\
\vdots \\
0
\end{array}\right] .
$$

Since the first column of $P$ is given by $1_{n}$,

$$
\lim _{t \rightarrow \infty} x(t)=\left[\begin{array}{c}
\chi_{1}(0) \\
\vdots \\
\chi_{1}(0)
\end{array}\right]=\left[\begin{array}{c}
P_{1 i}^{-1} x(0) \\
\vdots \\
P_{1 i}^{-1} x(0)
\end{array}\right],
$$

where $P_{1 i}^{-1}$ correspond to the first row of matrix $P^{-1}$.

Additionally, from (82), it is shown that states $x_{i}(t)$ for $i=1, \ldots, n$ converge to the same value

$$
x_{i}(\infty)=\chi_{i}(0)=P_{1 i}^{-1} x(0)
$$

that depends on the agents initial conditions.

The preceding developments allow formally stating the following result.

Theorem 8. Consider a group of $n$ single integrator agents with a fixed topology having a spanning tree as given in (9). Protocol (69) based on predicted states given by (23) solves the delayed consensus problem if and only if $\tau<\pi / 2 \lambda$. Moreover, $x_{i}(t) \rightarrow$ $P_{1 i}^{-1} x(0)$.

Remark 9. In fact it is not necessary that function $f(t)$ in (79b) tends exponentially to zero; it suffices its asymptotic convergence. The exponential property is important in order to improve the rate of convergence to the agreement of the agents.
5.2. Double Integrator Agents Case. From the definition of the advance variables (40) and the predictor-observer (44), it is possible to see that the future estimation of the state $\widehat{x}_{i j}(t+\tau)$ for $i=1, \ldots, n$ and $j=1,2$ is defined as

$$
\widehat{x}_{i j}(t+\tau)=\widehat{w}_{i j}(t) .
$$

In this case, the noncausal feedback (18) can be modified as

$$
\begin{aligned}
u_{i}(t)=-\sum_{j=1}^{n} a_{i j}\left[\left(\widehat{w}_{i 1}(t)-\widehat{w}_{j 1}(t)\right)\right. \\
\left.+\gamma\left(\widehat{w}_{i 2}(t)-\widehat{w}_{j 2}(t)\right)\right],
\end{aligned}
$$

which in compact form results

$$
u(t)=-L \widehat{w}_{1}(t)-\gamma L \widehat{w}_{2}(t),
$$

where $\widehat{w}_{j}=\left[\widehat{w}_{1 j}, \ldots, \widehat{w}_{n j}\right]^{T}, j=1,2$, and $L \in \mathbb{R}^{n \times n}$ is the Laplacian generated by the network topology.

Feedback (86), in closed-loop with system (12), produces

$$
\begin{aligned}
& \dot{x}_{1}(t)=x_{2}(t), \\
& \dot{x}_{2}(t)=-L \widehat{w}_{1}(t-\tau)-\gamma L \widehat{w}_{2}(t-\tau) .
\end{aligned}
$$

By considering definition (84), (43) can be expressed as $e_{w j}(t-\tau)=x_{j}(t)-\widehat{x}_{j}(t)$, for $j=1,2$, turning (87) in

$$
\begin{aligned}
\dot{x}_{1}(t)= & x_{2}(t), \\
\dot{x}_{2}(t)=- & L x_{1}(t)-\gamma L x_{2}(t) \\
& +L e_{w_{1}}(t-\tau)-\gamma L e_{w_{2}}(t-\tau) .
\end{aligned}
$$

The overall dynamic of the closed-loop (12)-(86) is obtained from (88) and (48), resulting in

$$
\begin{aligned}
& \dot{x}_{1}=x_{2}, \\
& \dot{x}_{2}=-L x_{1}-\gamma L x_{2}+L e_{w_{1}}(t-\tau)-\gamma L e_{w_{2}}(t-\tau), \\
& \dot{e}_{w_{1}}=e_{w_{2}}-\lambda_{1} e_{w_{1}}(t-\tau), \\
& \dot{e}_{w_{2}}=-\lambda_{0} e_{w_{1}}(t-\tau) .
\end{aligned}
$$

Notice that, regarding (40), system (89) can be rewritten in terms of the future variables, yielding the representation

$$
\begin{gathered}
\dot{w}_{1}=w_{2} \\
\dot{w}_{2}=-L w_{1}-\gamma L w_{2}+L e_{w_{1}}+\gamma L e_{w_{2}} \\
\dot{e}_{w_{1}}=e_{w_{2}}-\lambda_{1} e_{w_{1}}(t-\tau) \\
\dot{e}_{w_{2}}=-\lambda_{0} e_{w_{1}}(t-\tau)
\end{gathered}
$$

where $w_{j}=\left[w_{1 j}, \ldots, w_{n j}\right]^{T}$, for $j=1,2$.

Taking into account from (68) that subsystem (90b) is exponentially stable, it is clear that

$$
\left(L w_{1}, w_{2}\right)=(0,0)
$$


is an equilibrium point of the subsystem (90a). Notice that $L w_{1}=0$ is satisfied for $w_{11}=w_{21}=\cdots=w_{n 1}$, since the sum of the elements of each row of $L$ is always null. Notice also that the convergence of $w_{i 1}(t)=x_{i_{1}}(t+\tau)$ and $w_{i 2}(t)=x_{i_{2}}(t+\tau)$ implies the convergence of the states $x_{i_{1}}(t)$ and $x_{i_{2}}(t)$ due to the finite value of $\tau$.

5.3. Position and Velocity Convergence. The dynamic of system (90a) can be written alternatively by considering the transformation

$$
\left[\begin{array}{l}
w_{1} \\
w_{2}
\end{array}\right]=\left[\begin{array}{ll}
P & 0 \\
0 & P
\end{array}\right]\left[\begin{array}{l}
\varphi_{1} \\
\varphi_{2}
\end{array}\right]
$$

with matrix $P$ as in (76) yielding

$$
\begin{gathered}
\dot{\varphi}_{1}=\varphi_{2} \\
\dot{\varphi}_{2}=-P^{-1} L P \varphi_{1}-\gamma P^{-1} L P \varphi_{2} \\
+P^{-1} L e_{w_{1}}+\gamma P^{-1} L e_{w_{2}}
\end{gathered}
$$

Since $P^{-1} L P=J$, with $J$ as the Jordan form of $L$, then (93) can be rewritten as

$$
\dot{\varphi}=\Gamma_{J} \varphi+B_{L} e_{w}
$$

for $\varphi=\left[\varphi_{1}, \varphi_{2}\right]^{T}$ with $\varphi_{1}=\left[\begin{array}{lll}\varphi_{11} & \ldots & \varphi_{1 n}\end{array}\right]^{T}, \varphi_{1}=$ $\left[\begin{array}{lll}\varphi_{21} & \cdots & \varphi_{2 n}\end{array}\right]^{T}$, and

$$
\Gamma_{J}=\left[\begin{array}{cc}
0 & I \\
-J & -\gamma J
\end{array}\right], \quad B_{L}=\left[\begin{array}{cc}
0 & 0 \\
P^{-1} L & \gamma P^{-1} L
\end{array}\right] \text {. }
$$

The characteristic polynomial of the autonomous system

$$
\dot{\varphi}=\Gamma_{J} \varphi
$$

is obtained as

$$
\begin{aligned}
p_{\varphi}(s) & =\operatorname{det}\left\{s I-\Gamma_{J}\right\} \\
& =\operatorname{det}\left\{s^{2} I+\gamma J s+J\right\} \\
& =\operatorname{det}\left\{I s^{2}+(1+\gamma s) J\right\} \\
& =\operatorname{det}\left\{P^{-1}\left(I s^{2}+(1+\gamma s) L\right) P\right\} .
\end{aligned}
$$

By considering the complex Jordan form $J$ with eigenvalues $\mu_{i}, i=1, \ldots, n$, it is obtained that

$$
p_{\varphi}(s)=\operatorname{det}\left\{s I-\Gamma_{J}\right\}=\prod_{i=1}^{n}\left(s^{2}+(1+\gamma s) \mu_{i}\right)
$$

from where it is clear that each eigenvalue of $L$ produces two eigenvalues of $\Gamma_{J}[7]$.

Lemma 10. Suppose that $\mu_{i} \neq 0$ is an eigenvalue of the Laplacian matrix L associated with a communication topology having a spanning tree of a group of $n$ double integrators agents. Then, every $\mu_{i}$ will produce two eigenvalues $\mu_{J_{i}}$ of $\Gamma_{J}$ with $\operatorname{Re}\left\{\mu_{J_{i}}\right\}<0$ if and only if

$$
\gamma>\max _{\mu_{i} \neq 0} \frac{1}{\left|\mu_{i}\right|} \frac{\operatorname{Im}\left(\mu_{i}\right)}{\sqrt{\operatorname{Re}\left(\mu_{i}\right)}} .
$$

Proof. From (98), the eigenvalues of $\Gamma_{J}$ can be obtained from the equation

$$
s^{2}+\gamma \mu_{i} s+\mu_{i}=0
$$

for $i=1, \ldots, n-1$, in the form

$$
s_{1,2}=\frac{1}{2}\left[-\gamma \mu_{i} \pm \sqrt{\gamma^{2} \mu_{i}^{2}-4 \mu_{i}}\right] .
$$
that By considering that $\mu_{i}=a_{i}+j b_{i}$ with $j^{2}=-1$, it is obtained

$$
\begin{aligned}
2 s_{1,2}= & -\gamma a_{i}-j \gamma b_{i} \pm \sqrt{\gamma^{2}\left(a_{i}^{2}-b_{i}^{2}\right)-4 a_{i}+j\left(2 \gamma^{2} a_{i} b_{i}-4 b_{i}\right)} \\
= & -\gamma a_{i}-j \gamma b_{i} \\
& \pm\left[\frac{1}{\sqrt{2}} \sqrt{\sqrt{\left(\gamma^{2}\left(a_{i}^{2}-b_{i}^{2}\right)-4 a_{i}\right)^{2}+\left(2 \gamma^{2} a_{i} b_{i}-4 b_{i}\right)^{2}}+\gamma^{2}\left(a_{i}^{2}-b_{i}^{2}\right)-4 a_{i}}\right. \\
& +j \frac{\operatorname{sgn}\left(2 \gamma^{2} a_{i} b_{i}-4 b_{i}\right)}{\sqrt{2}} \\
& \left.\times \sqrt{\sqrt{\left(\gamma^{2}\left(a_{i}^{2}-b_{i}^{2}\right)-4 a_{i}\right)^{2}+\left(2 \gamma^{2} a_{i} b_{i}-4 b_{i}\right)^{2}}-\gamma^{2}\left(a_{i}^{2}-b_{i}^{2}\right)+4 a_{i}}\right] .
\end{aligned}
$$


Therefore, to assure $\operatorname{Re}\left\{\mu_{J_{i}}\right\}<0$, the following inequality is considered:

$$
-\gamma a_{i}+\frac{1}{\sqrt{2}} \sqrt{\sqrt{\left(\gamma^{2}\left(a_{i}^{2}-b_{i}^{2}\right)-4 a_{i}\right)^{2}+\left(2 \gamma^{2} a_{i} b_{i}-4 b_{i}\right)^{2}}+\gamma^{2}\left(a_{i}^{2}-b_{i}^{2}\right)-4 a_{i}}<0
$$

that produces

$$
\begin{aligned}
& {\left[2 \gamma^{2} a_{i}^{2}-\left(\gamma^{2}\left(a_{i}^{2}-b_{i}^{2}\right)-4 a_{i}\right)\right]^{2}} \\
& \quad>\left(\gamma^{2}\left(a_{i}^{2}-b_{i}^{2}\right)-4 a_{i}\right)^{2}+\left(2 \gamma^{2} a_{i} b_{i}-4 b_{i}\right)^{2} .
\end{aligned}
$$

After some manipulations, it is obtained that

$$
\gamma^{2}>\frac{b_{i}^{2}}{a_{i}} \frac{1}{a_{i}^{2}+b_{i}^{2}}
$$

This proves the lemma.

Remark 11. It should be noticed that Lemma 10 can be directly applied to the consensus for double integrator agents free of delay. In particular, Lemma 10 improves the bound reported on Lemma 4.4 and Theorem 4.2 in [7] providing necessary and sufficient conditions instead of only sufficient ones.

Based on Lemma 10, the solution to the consensus protocol for double integrator agents is stated as follows.

Theorem 12. Consider a group of $n$ double integrator agents with a fixed information topology having a spanning tree. Protocol (85) solves the consensus problem associated to double integrator agents (11), if and only if

$$
\gamma>\max _{\mu_{i} \neq 0} \frac{1}{\left|\mu_{i}\right|} \frac{\operatorname{Im}\left(\mu_{i}\right)}{\sqrt{\operatorname{Re}\left(\mu_{i}\right)}} .
$$

In particular $x_{1}(\infty)=1_{n}\left[T_{1 i}^{-1}+t T_{2 i}^{-1}\right] x(0)$ and $x_{2}(\infty)=$ $1_{n}\left[T_{2 i}^{-1}\right] x(0)$ for $T_{1 i}^{-1}$ and $T_{2 i}^{-1}$ defined, respectively, by the first and second row of the inverse of matrix $T=\left[t_{1}, \ldots, t_{2 n}\right]$, formed by the generalized eigenvectors $t_{i}$ of $\Gamma_{J}$.

Proof. Taking into account the structure of the matrix $J$ given in (76), (93) can be rewritten as

$$
\begin{aligned}
& \dot{\varphi}_{11}=\varphi_{21}, \\
& \dot{\varphi}_{21}=0, \\
& \dot{\varphi}_{1 b}=\varphi_{2 b}, \\
& \dot{\varphi}_{2 b}=-\bar{J} \varphi_{1 b}-\gamma \bar{J} \varphi_{2 b}+\bar{J} P^{-1} e_{w 1}+\gamma \bar{J} P^{-1} e_{w_{2}},
\end{aligned}
$$

where $\varphi_{11}, \varphi_{21} \in \mathbb{R}$ and $\varphi_{1 b}, \varphi_{2 b} \in \mathbb{R}^{n-1}$. From Lemma 10, subsystem (107b) is exponentially stable with an exogenous input $f(t)=\bar{J} P^{-1} e_{w 1}+\gamma \bar{J} P^{-1} e_{w_{2}}$. Therefore, since $\lim _{t \rightarrow \infty} f(t)=0$, the state convergence of system (107a) and (107b) is obtained by considering the response of the autonomous system

$$
\begin{aligned}
& {\left[\begin{array}{c}
\dot{\varphi}_{11} \\
\dot{\varphi}_{21} \\
\dot{\varphi}_{1 b} \\
\dot{\varphi}_{2 b}
\end{array}\right] }=\left[\begin{array}{cc|c}
0 & 1 & 0_{2 \times(2 n-2)} \\
0 & 0 & \multicolumn{2}{|c}{}
\end{array}\right]\left[\begin{array}{l}
\varphi_{11} \\
\varphi_{21} \\
\varphi_{1 b} \\
0_{(2 n-2) \times 2}
\end{array}\right] \\
&\left.\begin{array}{c}
0_{(n-1) \times(n-1)} \\
\varphi_{(n-1) \times(n-1)}
\end{array}\right] \\
& A_{\varphi}\left[\begin{array}{c}
\varphi_{11} \\
\varphi_{21} \\
\varphi_{1 b} \\
\varphi_{2 b}
\end{array}\right] .
\end{aligned}
$$

Define now a transformation matrix $T$ formed by the generalized eigenvectors $t_{1}, t_{2}, \ldots, t_{2 n}$ of $\Gamma_{J} ; T=\left[t_{1}, \ldots, t_{2 n}\right]$. Notice that the first diagonal block of $A_{\varphi}$ is already the Jordan block associated with the pair of zero eigenvalues $\mu_{1}=\mu_{2}=0$ of $A_{\varphi}$. By the uniqueness of the Jordan canonical form, up to the order of the eigenvalues, the corresponding eigenvectors are given by the first and $(n+1)$ th columns of the matrix considered in transformation (92) given as [7]

$$
t_{1}=\left[\begin{array}{l}
1_{n} \\
0_{n}
\end{array}\right], \quad t_{2}=\left[\begin{array}{l}
0_{n} \\
1_{n}
\end{array}\right] .
$$

The remaining eigenvectors, $t_{3}, \ldots, t_{2 n}$, will transform the second block of $A_{\varphi}$ to the Jordan form. Therefore, by defining the transformation $w=T \psi$, the equivalent autonomous system is obtained:

$$
\dot{\psi}=J_{T} \psi
$$

for $\psi=\left[\psi_{1}, \psi_{2}\right]^{T}$ with $\psi_{1}=\left[\psi_{11}, \psi_{12}\right]^{T}, \psi_{2}=$ $\left[\psi_{21}, \ldots, \psi_{2(2 n-2)}\right]^{T}$, and

$$
J_{T}=T^{-1} \Gamma T \text {. }
$$

In this case, $J_{T}$ takes the form

$$
J_{T}=\left[\begin{array}{cc|c}
0 & 1 & 0_{2 \times(2 n-2)} \\
0 & 0 & \bar{J}_{T}
\end{array}\right]
$$

with $\bar{J}_{T} \in \mathbb{R}^{(2 n-2) \times(2 n-2)}$ in a Jordan block. The time solution of (110) can be easily obtained as

$$
\psi(t)=e^{J_{T} t} \psi(0)
$$


from where the evolution of the future state can be obtained in the form

$$
w(t)=T e^{J_{T} t} \psi(0) .
$$
to

By considering (112), the future states converge according

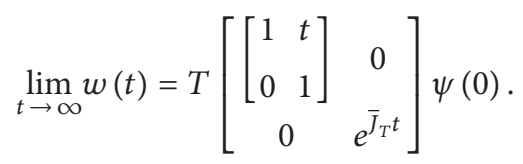

Notice that since all the eigenvalues associated with $\bar{J}_{T}$ are negative, the following is obtained:

$$
\lim _{t \rightarrow \infty} w(t)=T\left[\begin{array}{c}
\psi_{11}(0)+t \psi_{21}(0) \\
\psi_{21}(0) \\
0 \\
\vdots \\
0
\end{array}\right],
$$

in addition, the general structure of $T$ allows writing

$$
\begin{aligned}
w(\infty) & =\left[\begin{array}{lll}
1_{n} & 0_{n} & * \\
0_{n} & 1_{n} & *
\end{array}\right]\left[\begin{array}{c}
\psi_{11}(0)+t \psi_{21}(0) \\
\psi_{21}(0) \\
0 \\
\vdots \\
0
\end{array}\right] \\
& =\left[\begin{array}{c}
1_{n}\left(\psi_{11}(0)+t \psi_{21}(0)\right) \\
1_{n}\left(\psi_{21}(0)\right)
\end{array}\right] .
\end{aligned}
$$

Since $\psi=T^{-1} w$,

$$
\begin{gathered}
w_{1}(\infty)=1_{n}\left[T_{1 i}^{-1}+t T_{2 i}^{-1}\right] w(0), \\
w_{2}(\infty)=1_{n}\left[T_{2 i}^{-1}\right] w(0),
\end{gathered}
$$

where $T_{1 i}^{-1}$ and $T_{2 i}^{-1}$ represent, respectively, the first and second row of matrix $T^{-1}$.

To complete the proof, notice that, due to relation (40), a time shift of $\tau$ units of time provides

$$
\begin{aligned}
& x_{1}(\infty)=1_{n}\left[T_{1 i}^{-1}+t T_{2 i}^{-1}\right] x(0), \\
& x_{2}(\infty)=1_{n}\left[T_{2 i}^{-1}\right] x(0) .
\end{aligned}
$$

The computation of the final values given in (119) can be simplified by obtaining $T^{-1}$ by means of a set on $n$ independent left eigenvectors $h_{i}, i=1, \ldots, 2 n$ of $T$. It has been proved in [7] that $h_{1}=\left[\begin{array}{ll}p^{T} & 0_{n}^{T}\end{array}\right]^{T}$ and $h_{2}=\left[\begin{array}{ll}0_{n}^{T} & p^{T}\end{array}\right]^{T}$ are the left eigenvectors associated with the zero eigenvalue

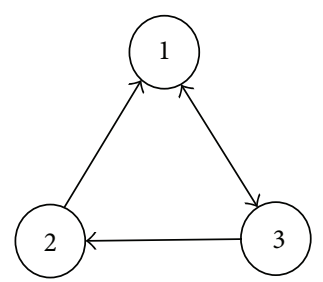

FIGURE 1: Three single integrator agents topology.

with $p$ being a vector that satisfies $p^{T} L=0$ and $P^{T} 1_{n}=1$ for $P$ as in (73). A direct computation of $h_{i}$ and $h_{2}$ can be done by considering $p^{T} L=p^{T} L P=0$ which implies that

$$
p^{T} P J=0 .
$$

Since $P=\left[v_{1}, \ldots, v_{n}\right]$ with $v_{1}=1_{n}$, then $p^{T} v_{1}=1$. By the structure of matrix $J$ and condition (120), it is obtained that $p^{T} v_{i}=0$ for $i=2, \ldots, n$. It is clear now that $p^{T}$ correspond to the first row of matrix $P^{-1}$ denoted in Theorem 8 as $P_{1 i}^{-1}$. Direct computations show that

$$
\begin{aligned}
& x_{1}(\infty)=1_{n} P_{1 i}^{-1}\left[x_{1}(0)+t x_{2}(0)\right], \\
& x_{2}(\infty)=1_{n} P_{1 i}^{-1} x_{2}(0) .
\end{aligned}
$$

Therefore, the knowledge of matrices $L$ and $P$ gives the information to compute the value of the agreement.

\section{Numeric Evaluation}

To show the effectiveness of the prediction-based solution of the delayed consensus problem, two numerical problems will be considered.

6.1. Example 1. To compare the proposed solution with a previous result, this example is inspired from [9], where three agents with the topology depicted in Figure 1 are considered. The associated Laplacian matrix takes the form

$$
L=\left[\begin{array}{ccc}
1 & 0 & -1 \\
-1 & 1 & 0 \\
-1 & -1 & 2
\end{array}\right]
$$

where $\lambda_{\max }(L)=2$. Following the control strategy presented in [9], the delayed consensus problem can be solved for $\tau<$ $\pi / 2 \lambda_{\max }(L)=0.785 \mathrm{~s}$.

When applying the prediction-based strategy proposed in this work, it can be shown that by considering the design parameter $\lambda=1.0472$ a maximum time-delay $\tau^{*}=$ $1.5 \mathrm{~s}$ is obtained. For this experiment, $\tau=0.785$ and the initial conditions $x(0)=[-4,1,3]^{T}$ are considered. The convergence to zero of the observation error $e_{w}(t-\tau)=x(t)-$ $\widehat{x}(t)$ is shown in Figure 2(a) while the consensus of the agents state is shown in Figure 2(b). The evolution of the control signals used on the experiment is depicted in Figure 2(c). 


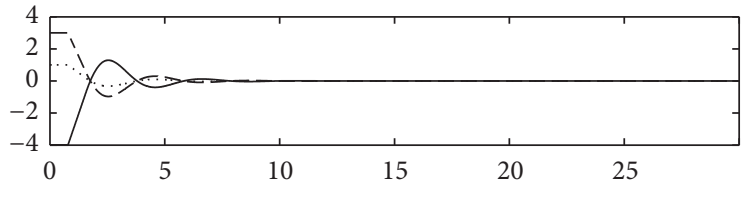

$t$

$-e_{1}(t-\tau)$

$\ldots e_{2}(t-\tau)$

$---e_{3}(t-\tau)$

(a) Observation-prediction error $e_{\omega}(t-\tau)$

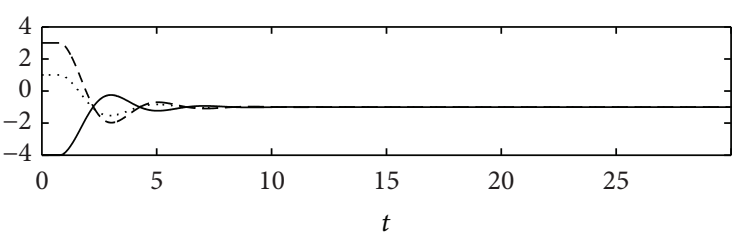

$-x_{1}(t)$

$\ldots \ldots x_{2}(t)$

$--x_{3}(t)$

(b) System output $x(t)$

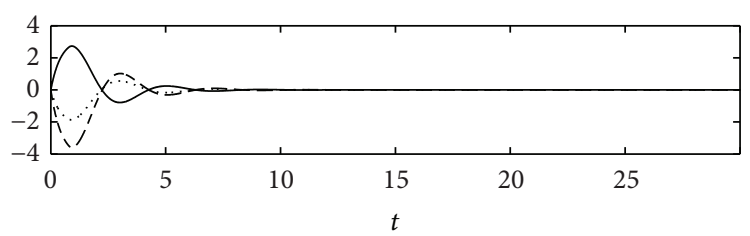

$-u_{1}(t)$

$\ldots u_{2}(t)$

$---u_{3}(t)$

(c) Control signal $u(t)$

FIGURE 2: Single integrator case, $\tau=0.785 \mathrm{~s}$.
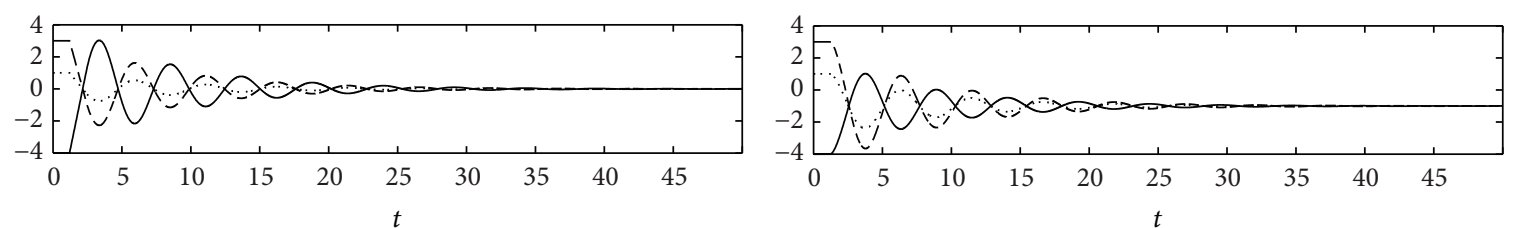

$\begin{array}{ll}- & e_{1}(t-\tau) \\ \ldots \ldots & e_{2}(t-\tau) \\ --- & e_{3}(t-\tau)\end{array}$

$-x_{1}(t)$

....... $x_{2}(t)$

$--x_{3}(t)$

(b) System output $x(t)$

(a) Observation-prediction error $e_{\omega}(t-\tau)$

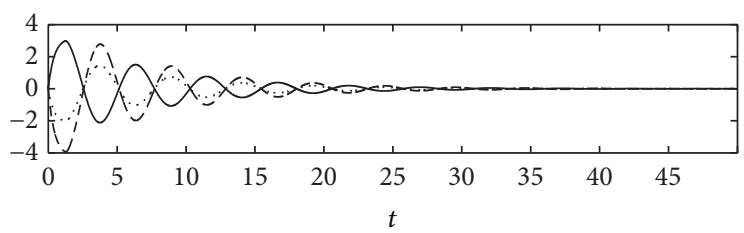

$\begin{array}{ll} & u_{1}(t) \\ \ldots \ldots & u_{2}(t) \\ -\ldots & u_{3}(t)\end{array}$

(c) Control signal $u(t)$

FIGURE 3: Single integrator case, $\tau=1.2 \mathrm{~s}$.

In a more challenging situation, where the time-delay is set as $\tau=1.2 \mathrm{~s}$, Figure 3(a) shows the time evolution of the delayed prediction error. Figure 3(b) depicts the evolution of the states and the corresponding control signals are shown in Figure 3(c). Notice how the rate of convergence for this case is slower than the previous one due to the fact that the delay is near the maximum allowable delay time.
To determine the convergence value of the agents' state, notice that matrix $P$ given in (73) takes the form

$$
P=\left[\begin{array}{ccc}
1 & -\frac{1}{2} & -\frac{1}{4} \\
1 & \frac{1}{2} & -\frac{1}{4} \\
1 & \frac{1}{2} & \frac{3}{4}
\end{array}\right] .
$$




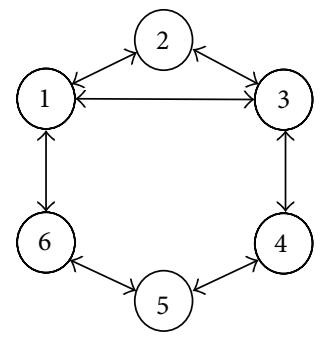

FIGURE 4: Topology for the group of double integrator agents.

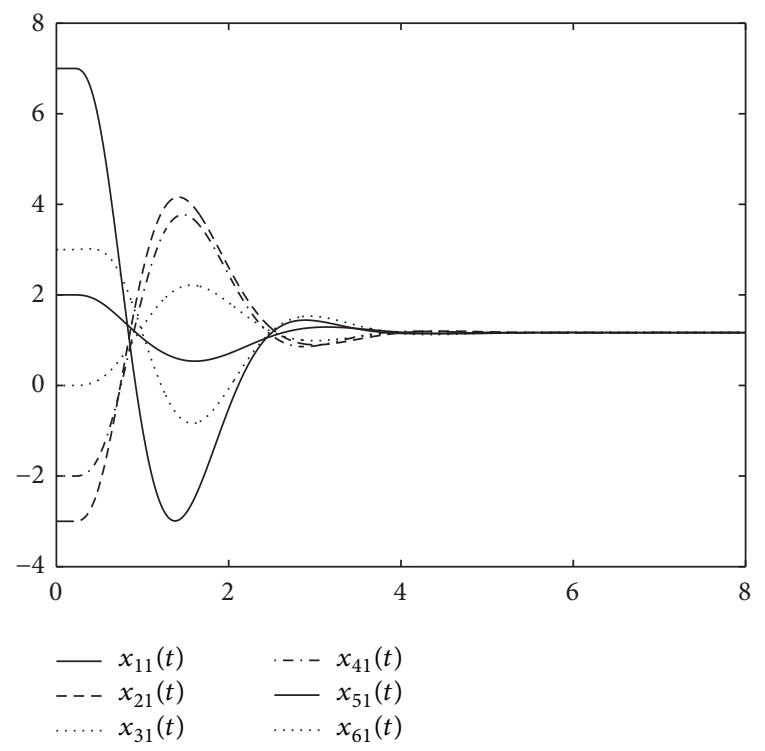

FIGURE 5: Double integrator case and time position evolution.

Therefore, by considering $(82), x_{i}(t) \rightarrow P_{1 i}^{-1} x(0)$; that is,

$$
P_{1 i}^{-1} x(0)=\left[\begin{array}{lll}
0.5 & 0.25 & 0.25
\end{array}\right]\left[\begin{array}{lll}
-4 & 1 & 3
\end{array}\right]^{T}=-1 .
$$

6.2. Example 2. In this example, a group of double integrator agents as the one in (11) is considered. The topology of the group of agents is shown in Figure 4 that was taken from [26]. The Laplacian matrix is given as

$$
L=\left[\begin{array}{cccccc}
3 & -1 & -1 & 0 & 0 & -1 \\
-1 & 2 & -1 & 0 & 0 & 0 \\
-1 & -1 & 3 & -1 & 0 & 0 \\
0 & 0 & -1 & 2 & -1 & 0 \\
0 & 0 & 0 & -1 & 2 & -1 \\
-1 & 0 & 0 & 0 & -1 & 2
\end{array}\right]
$$

with eigenvalues $\{0,1,3,4,3+\sqrt{2}, 3-\sqrt{2}\}$. The experiment was carried out by considering the initial conditions $x_{11}(0)=$ $7, x_{21}(0)=-3, x_{31}(0)=0, x_{41}(0)=-2, x_{51}(0)=2$, $x_{61}(0)=3$, and $x_{i 2}(0)=0$ for $i=1, \ldots, 6$. For the design of the observer $\lambda_{0}=0.5$ and $\lambda_{1}=1$ were considered while for the feedback law $\gamma=1$ was chosen.

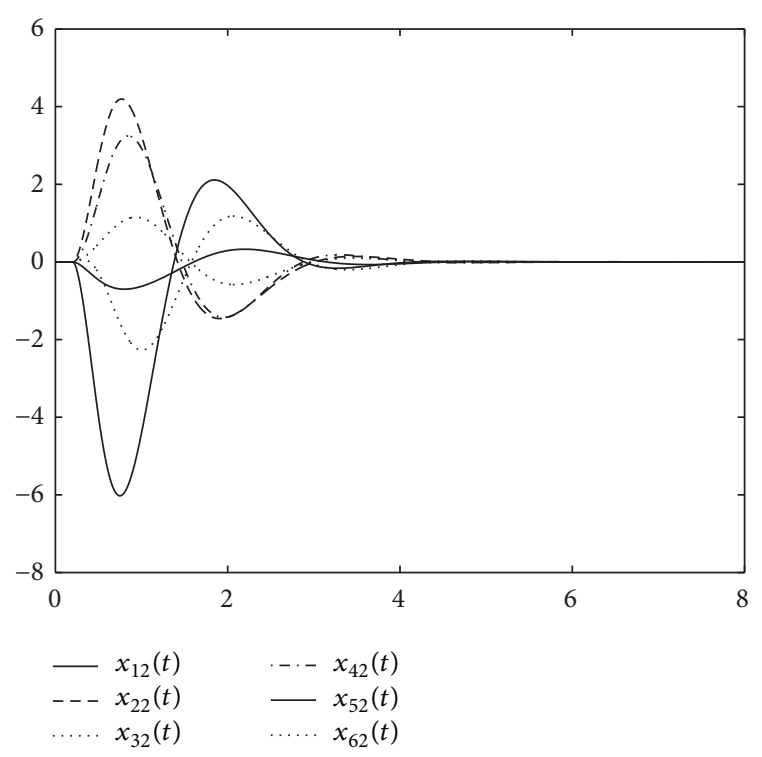

FiguRE 6: Double integrator case and time velocity evolution.

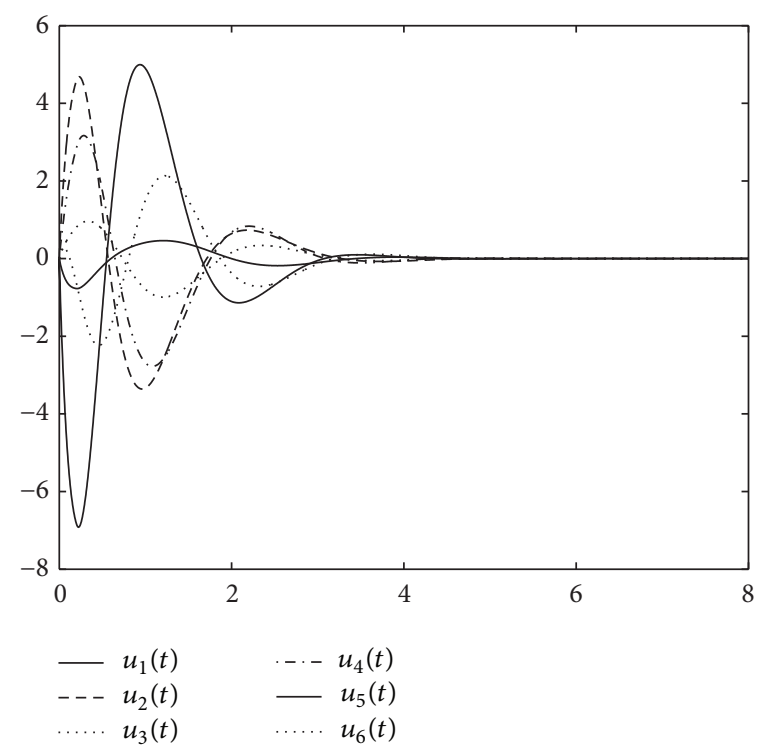

FIgURE 7: Double integrator case and control signals.

According to the result presented in [26], the maximum time-delay supported for the control scheme is $\tau^{*}=0.2293$. To show the superiority of the solution presented in this work, a time-delay $\tau=0.5 \mathrm{~s}$ is considered that cannot be handled by the result in [26]. The prediction-based solution proposed in this work produces the position time evolution $x_{i 1}$ described in Figure 5 and the velocity evolution $x_{i 2}$ presented in Figure 6. These two figures show clearly how the consensus problem is solved. The time evolution of the control signal is shown in Figure 7. Since $T_{1 i}^{-1}=\left[P_{1 i}^{-1}, 0_{n}^{T}\right]$ and $T_{2 i}^{-1}=\left[0_{n}^{T}, P_{1 i}^{-1}\right]$ with $P_{1 i}^{-1}=(1 / 6) 1_{n}^{T}$, from Theorem 12, the convergence values are $x_{1}(\infty)=(7 / 6) 1_{n}$ and $x_{2}(\infty)=0_{n}$. 


\section{Conclusions}

The delayed consensus problem is analyzed for a group of agents constituted by single and double integrators, subject to constant time-delay communication effects. To solve this problem, a protocol based on a predictor-observer scheme is proposed that allows getting estimated future values of the states of the agents that are used on the protocols that solve the consensus problems improving previously reported results. The proposed scheme has the advantage that the solution conditions depend on design parameters and are not related to the maximum eigenvalue of the Laplacian matrix associated with the considered topology as previous results. An adequate performance of the proposed solutions by means of numerical simulations that take into account time-delays values that cannot be handled by the previous solution of the problem is shown.

\section{Conflict of Interests}

The authors declare that there is no conflict of interests regarding the publication of this paper.

\section{References}

[1] W. Ren and R. W. Beard, "Trajectory tracking for unmanned air vehicles with velocity and heading rate constraints," IEEE Transactions on Control Systems Technology, vol. 12, no. 5, pp. 706-716, 2004.

[2] J. R. Carpenter, "Decentralized control of satellite formations," International Journal of Robust and Nonlinear Control, vol. 12, no. 2-3, pp. 141-161, 2002.

[3] J. A. Fax and R. M. Murray, "Information flow and cooperative control of vehicle formations," IEEE Transactions on Automatic Control, vol. 49, no. 9, pp. 1465-1476, 2004.

[4] R. Olfati-Saber and R. M. Murray, "Distributed cooperative control of multiple vehicle formations using structural potential functions," in Proceedings of the 15th IFAC Triennial World Congress, 2002.

[5] R. Vidal, O. Shakernia, and S. Sastry, "Formation control of nonholonomic mobile robots with omnidirectional visual servoing and motion segmentation," in Proceedings of the IEEE International Conference on Robotics and Automation, vol. 1, pp. 584-589, Taipei, Taiwan, September 2003.

[6] W. Ren, R. W. Beard, and E. M. Atkins, "A survey of consensus problems in multi-agent coordination," in Proceedings of the American Control Conference (ACC '05), pp. 1859-1864, IEEE, June 2005.

[7] W. Ren and E. Atkins, "Distributed multi-vehicle coordinated control via local information exchange," International Journal of Robust and Nonlinear Control, vol. 17, no. 10-11, pp. 1002-1033, 2007.

[8] W. Ren, R. W. Beard, and E. M. Atkins, "Information consensus in multivehicle cooperative control," IEEE Control Systems Magazine, vol. 27, no. 2, pp. 71-82, 2007.

[9] R. Olfati-Saber and R. M. Murray, "Consensus problems in networks of agents with switching topology and time-delays," IEEE Transactions on Transactions on Automatic Control, vol. 49, no. 9, pp. 1520-1533, 2004.

[10] P.-A. Bliman and G. Ferrari-Trecate, "Average consensus problems in networks of agents with delayed communications," in Proceedings of the 44th IEEE Conference on Decision and Control, and the European Control Conference (CDC-ECC '05), pp. 7066-7071, December 2005.

[11] T. Namerikawa and C. Yoshioka, "Consensus control of observer-based multi-agent system with communication delay," in Proceedings of the SICE Annual Conference, pp. 2414-2419, IEEE, Tokyo, Japan, August 2008.

[12] S. Skogestad, "Simple analitic rules for model reduction and pid controller tuning," Journal of Process Control, vol. 13, no. 4, pp. 291-309, 2003.

[13] O. J. M. Smith, "Closer control of loops with deadtime," Chemical Engineering Progress, vol. 53, no. 5, pp. 217-219, 1957.

[14] Z. J. Palmor, "Time-delay compensation smith predictor and its modifications," in The Control Handbook, pp. 224-237, CRC Press, 1996.

[15] A. Seuret, D. V. Dimarogonas, and K. H. Johansson, "Consensus under communication delays," in Proceedings of the 47th IEEE Conference on Decision and Control (CDC '08), pp. 4922-4927, IEEE, Cancún, Mexico, December 2008.

[16] W. Ren and R. W. Beard, Distributed Consensus in Multi-Vehicle Cooperative Control: Theory and Applications, Springer, 2007.

[17] W. Ren and R. W. Beard, "Consensus algorithms for doubleintegrator dynamics," in Distributed Consensus in Multi-vehicle Cooperative Control: Theory and Applications, Communications and Control Engineering, pp. 77-104, Springer, London, UK, 2008.

[18] W. Ren, "On consensus algorithms for double-integrator dynamics," IEEE Transactions on Automatic Control, vol. 53, no. 6, pp. 1503-1509, 2008.

[19] L. Moreau, "Stability of continuous-time distributed consensus algorithms," in Proceedings of the 43rd IEEE Conference on Decision and Control (CDC '04), pp. 3998-4003, December 2004.

[20] D. Lee and M. W. Spong, "Agreement with non-uniform information delays," in Proceedings of the IEEE American Control Conference, p. 6, Minneapolis, Minn, USA, June 2006.

[21] P. Lin and Y. Jia, "Consensus of a class of second-order multiagent systems with time-delay and jointly-connected topologies," IEEE Transactions on Automatic Control, vol. 55, no. 3, pp. 778-784, 2010.

[22] P. Lin and Y. Jia, "Consensus of second-order discrete-time multi-agent systems with nonuniform time-delays and dynamically changing topologies," Automatica, vol. 45, no. 9, pp. 21542158, 2009.

[23] Z. Yan, D. Wu, and Y. Liu, "Consensus of discrete multiagent system with various time delays and environmental disturbances," Entropy, vol. 16, no. 12, pp. 6524-6538, 2014.

[24] W. Zhu, "Consensus of discrete time second-order multiagent systems with time delay," Discrete Dynamics in Nature and Society, vol. 2012, Article ID 390691, 9 pages, 2012.

[25] Y. Chen, J. Lü, X. Yu, and Z. Lin, "Consensus of discrete-time second-order multiagent systems based on infinite products of general stochastic matrices," SIAM Journal on Control and Optimization, vol. 51, no. 4, pp. 3274-3301, 2013.

[26] L. Peng, J. Yingmin, D. Junping, and Y. Shiying, "Distributed consensus control for second-order agents with fixed topology and time-delay," in Proceedings of the 26th Chinese Control Conference (CCC '07), pp. 577-581, Hunan, China, July 2007.

[27] Y. Jiang, J. Liu, and S. Wang, "Robust integral sliding-mode consensus tracking for multi-agent systems with time-varying delay," Asian Journal of Control, 2014. 
[28] Z. Wen-Guang, L. Ji-Zhen, Z. De-Liang, and H. Yong, "Consensus of second-order multi-agent systems with nonuniform time delays," Chinese Physics B, vol. 22, no. 5, Article ID 050511, 2013.

[29] J. Liang-Hao and L. Xiao-Feng, "Consensus problems of firstorder dynamic multi-agent systems with multiple time delays," Chinese Physics B, vol. 22, no. 4, Article ID 040203, 6 pages, 2013.

[30] D. H. Wang, J. Yuan, J. Xu, and Z. H. Zhou, "An optimal consensus control for multiple agents system with time-delay and disturbances," Applied Mechanics and Materials, vol. 490491, pp. 828-831, 2014.

[31] H. Liu, G. Xie, and L. Wang, "Consensus of multi-agent systems with time-varying delay," in Proceedings of the 49th IEEE Conference on Decision and Control (CDC '10), pp. 3078-3083, IEEE, Atlanta, Ga, USA, December 2010.

[32] C. L. P. Chen, G.-X. Wen, Y.-J. Liu, and F.-Y. Wang, "Adaptive consensus control for a class of nonlinear multiagent time-delay systems using neural networks," IEEE Transactions on Neural Networks and Learning Systems, vol. 25, no. 6, pp. 1217-1226, 2014.

[33] C. Godsil and G. Royle, Algebraic Graph Theory, vol. 207 of Graduate Texts in Mathematics, Springer, Berlin, Germany, 2001.

[34] W. Ren and Y. Cao, Distributed Coordination of Multi-agent Networks: Emergent Problems, Models, and Issues, Springer, 2011.

[35] V. L. Kharitonov, Time-Delay Systems Lyapunov Functionals and Matrices, Birkhäuser, Boston, Mass, USA, 2010.

[36] S.-I. Niculescu, Delay Effects on Stability: A Robust Control Approach, Springer, London, UK, 2001.

[37] L. Perko, Differential Equations and Dynamical Systems, Texts in Applied Mathematics, Springer, New York, NY, USA, 1991. 


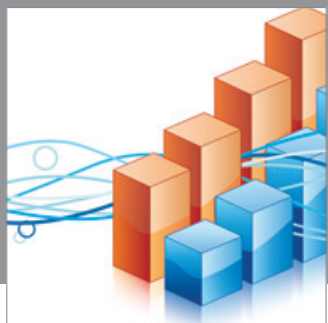

Advances in

Operations Research

mansans

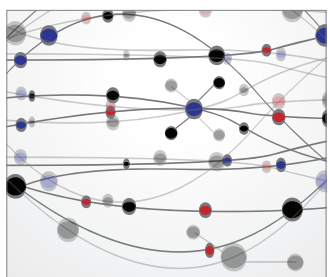

The Scientific World Journal
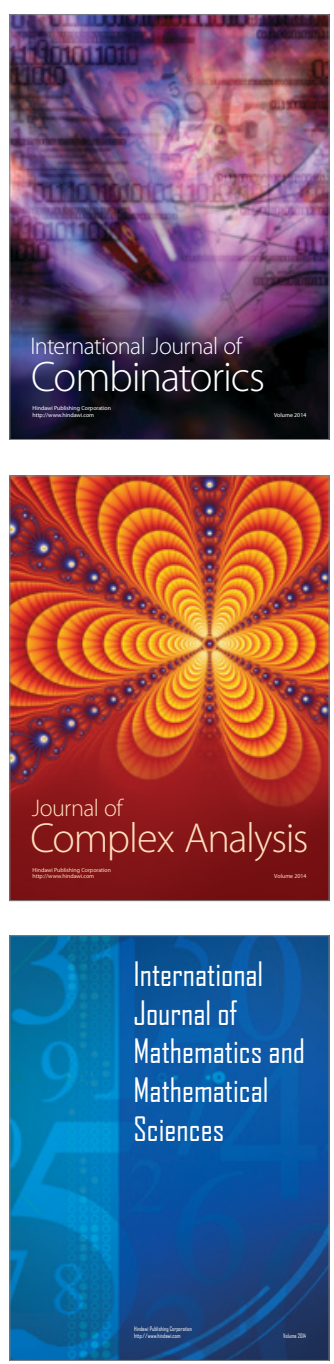
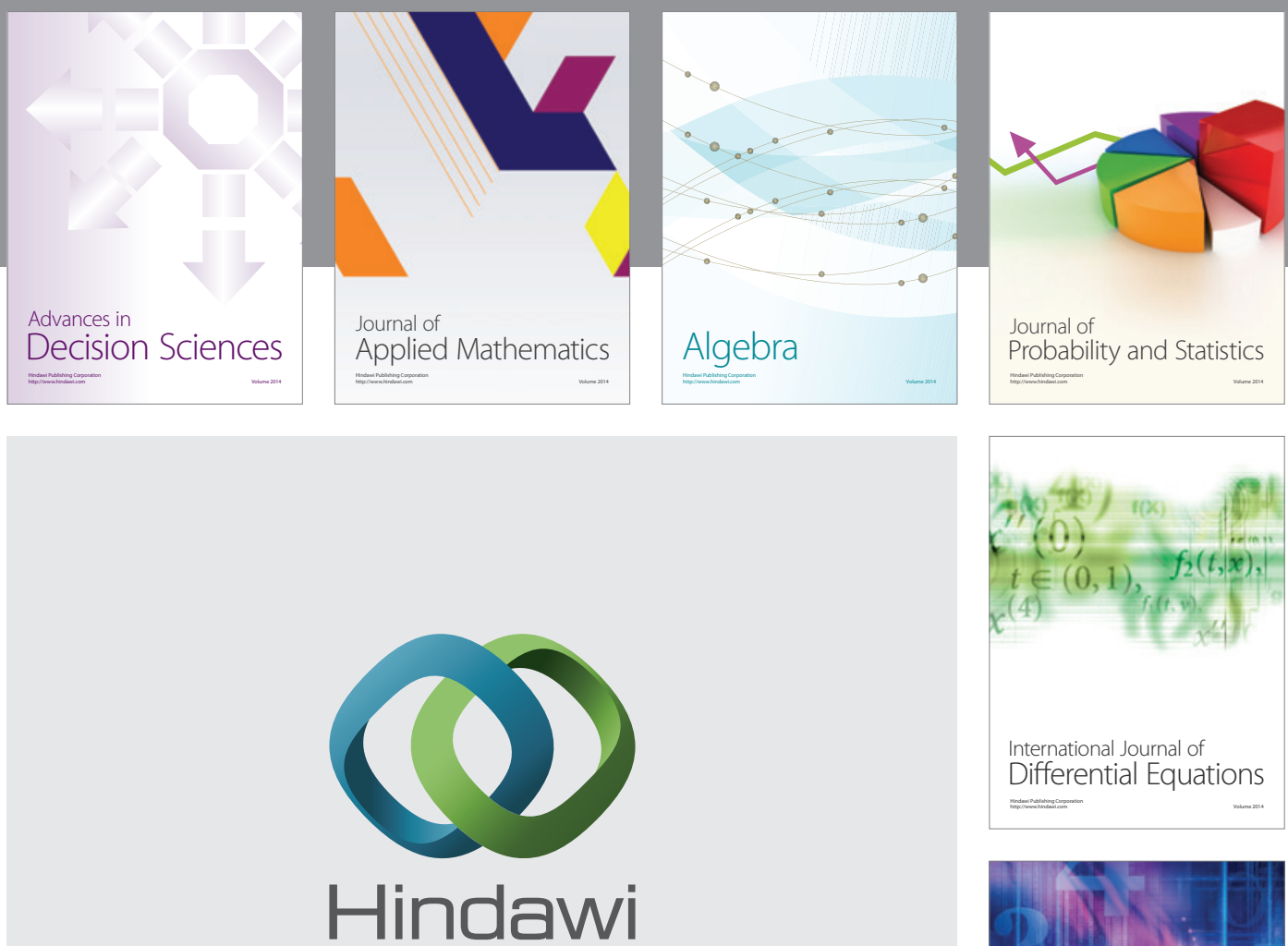

Submit your manuscripts at http://www.hindawi.com
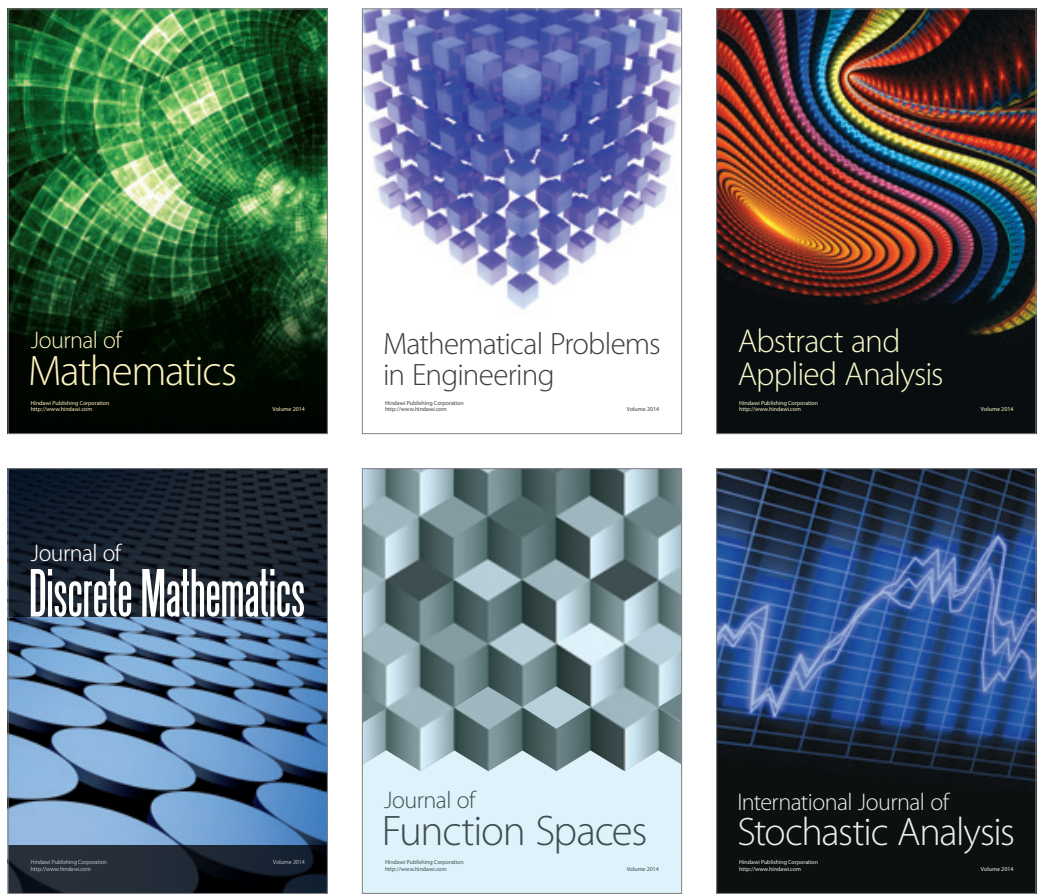

Journal of

Function Spaces

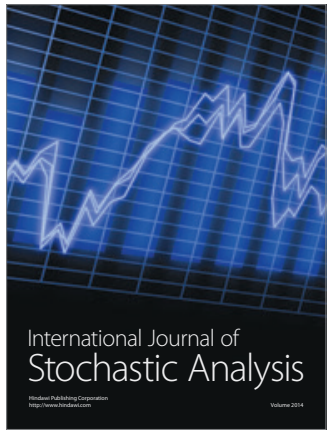

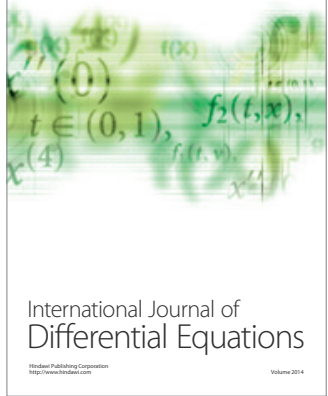
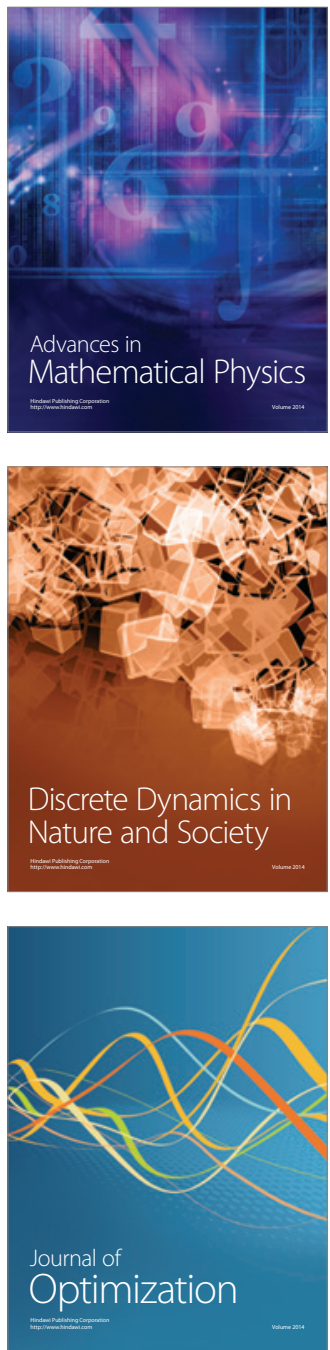